\title{
Index rerum ad Vol. 10
}

Accommodation for the elderly, 74

Activity level, 157

Adrenal and hypothalamic-pituitary

function, 334 Advances in stereoencephalotomy (B),

376 Amputee, lower limb, 321 Anaemia, 228 Anstaltsneurologie (B), 192 Architectural view of life in homes, 88 Ascites and hypothyroidism, 184 Auskultation des Herzen (B), 192

Blood pressure in old age, 37 Bromsulphthaleinretention in der Leber, 43

Capacity and age, 129, 146, 157 Chronic vitamin deficiency, 309 Chronische Nervenkrankheiten (B), 192 Clinical use of deoxyribonuclease, 116 Clinics, health centres and geriatrics, 299 Community care of the frail and elderly,

\section{5,358}

Death, attitudes to, 304 Degenerative skin disorders, 201 Deoxyribonuclease in clinical use, 116

Diagnosis of stroke, 174 Drugs and prescription, 271 D-Thyroxine, application thérapeu-tique, 1 Electrolyte transport, intra-cellular (B),

376 Encephalotomy (B), 376

Fe in serum, 21 Femur, fracture of, 257 Fracture of female femur, 257 Funktionsveränderungen der Leber, 43

Geriatric bed requirement in mental hospital, 30

- $\quad$ and psychological care, 65, 81

Geriatrics, teaching, 108

Glaucoma (B), 377

Hämodynamik und Auskultation (B), 192

Health centres and geriatrics, 299

Heparin treatment of macular degeneration, 288

Herz, Auskultation (B), 192

Homes, socio-architectural view of life, 88

Housing of the elderly, 187

Human plasma, water content, 193

- $\quad$ relations (B), 377

Hypokalaemia, 102

Hypothalamic-pituitary-adrenal func

tion, 334

Hypothyroidism and ascites, 184

Incontinence, urinary, 242, 345 Infection of urinary tract, 58, 242, 345 Intellectual performance, 157 Intra-cellular electrolyte transport (B),

376 Iron in serum, 21

Leber, Funktionsveränderungen, 43

Limb amputee, 321

Local health centres and geriatrics, 299 
Macular degeneration and heparin

treatment, 288 Medical aspects of accommodation for

the elderly, 74

ageing, 134

Mental disease, 293

- $\quad$ hospital, bed requirement, 30

380

Index rerum

Neurological aspects of ageing, 146 Neurology in an institution (B), 192 Nonagenarians and skeletal rarefaction, 348

Oral temperature of elderly, 281

Physical health, 157 Pituitary-adrenal and hypothalamic

function, 334 Plasma water content, 193 Population and urinary tract infection,

58, 242, 345 Prescription and drugs, 271 Proliferative skin disorders, 201 Psychiatric aspects of ageing, 139 Psycho-geriatric care, 65, 81

Rarefaction, skeletal, 348 Relations, human (B), 377

Senile macular degeneration, 288 Sensitivity to tuberculin, evolution of,

369 Serum iron in old patients, 21

Skeletal rarefaction, 348

Skin disorders, 201

Socio-architectural view of life in homes,

88 Stereoencephalotomy (B), 376 Stroke diagnosis, 174 Symposium on age and capacity, 129

Teaching geriatrics, 108 Temperature of elderly, 281 Thyroxine-D, application thérapeu-

tique, 1 Treatment of urinary tract infection, 58,

345 Tuberculin sensitivity, evolution of, 369

Urinary incontinence, 242, 345 - tract infection, 58, 242, 345

Vitamin deficiency, 309

Water content of human plasma, 193 Welfare service, temperatures of applicants for, 281 